\title{
A Descriptive Time Inventory of Emergency Department Nursing Workflows
}

\author{
Dana Skold, Alice Mitchell MD \\ Indiana University School of Medicine, Department of Emergency Medicine
}

Background: Previous emergency department (ED) process improvement efforts used probabilistic analytical or simulated models without considering the impact of specific tasks on ED patient flow and resource needs. We focus on the tasks and workflows that comprise nursing activity in an urban academic ED and level I trauma center receiving over 80,000 annual visits.

Experimental Design: Using a time/motion observational methodology, we create a minute-byminute time inventory account of nursing tasks and workflows as observed through the activities of 35 nurses over 124.5 hours, representative of 24/7 patient care in 7 ED care areas. "Tasks" were defined as discrete, measurable, and consequential step(s) to accomplish a clinically meaningful goal ("workflow"). The task with highest cognitive demand for each minute of observation was recorded. We also tracked 12 discrete highest-acuity ("shock") events and catalogued second-by-second observational accounts of each nurse diverted.

Results: Our data demonstrate significant variation in tasks based on time of day. We observed substantial operational load moving patients between care areas, with intake and discharge comprising $25 \%$ of nurse workflows. Downtime averaged $32 \%$, with variation depending on care area. Downtime was highest $(47 \%)$ with passive video monitoring of psychiatric care and lowest $(22 \%)$ in high-turnover intake areas. Highest-acuity patient-care events result in significant and variable nurse diversion from other tasks averaging 1:03:29 in combined nursing effort.

Conclusion and Potential Impact: Movement of patients between care areas represents significant operational load. Interruptions and task preparation accounted for a surprising portion of activity. Highest-acuity patient care events result in substantial and variable diversion of nurse care. 